\title{
A CONSTITUTIVE MODEL FOR RUBBLE MASONRY ALLOWING FOR SPREAD MICRO-CRACKS AND LOCALIZED MACRO-CRACKS
}

\author{
MANUELA A. SCAMARDO ${ }^{1 *}$, ALBERTO FRANCHI ${ }^{2}$ AND PIETRO G. CRESPI ${ }^{2}$ \\ ${ }^{1}$ Department of Architecture, Built Environment and Construction Engineering \\ Politecnico di Milano \\ Piazza Leonardo da Vinci 32, 20133 Milano, Italy \\ e-mail: manuela.scamardo@polimi.it (*corresponding author) \\ ${ }^{2}$ Department of Architecture, Built Environment and Construction Engineering \\ Politecnico di Milano \\ Piazza Leonardo da Vinci 32, 20133 Milano, Italy \\ email: \{alberto.franchi, pietro.crespi\}@polimi.it
}

Keywords: Masonry, Cracking, Plasticity, Softening, Finite Elements, PLCP

\begin{abstract}
Starting from a discussion on the experimental results obtained from diagonal compression tests executed on in-situ masonry panels, the paper presents a constitutive model, together with a numerical formulation, to describe the cracking phenomena in rubble masonry structures. A classical finite element discretization is assumed with the hypothesis of a homogenous continuum material. The adopted constitutive model identifies three different phases: (i) the elastic phase; (ii) the micro-cracking phase, in which the formation of microcracks, spread in the structural members, is accounted assuming a plastic material with a strain hardening stable behavior; (iii) the macro-cracks phase, in which the formation of macrocracks, developing along the edges of finite elements, are simulated by means of localized softening plastic deformation. While the numerical description of spread plasticity in the finite element framework is a topic that has been widely addressed in the past, the representation of localized plastic deformation and its implementation in a finite element code is an original contribution of the authors. From a computational point of view, the value of plastic deformations (i.e. crack openings) is found by solving a parametric linear complementarity problem (LCP) using mathematical programming algorithms. The main advantage of using an LCP method is its ability to deal also with configurations in which instability and a multiplicity of solutions are possible (e.g. softening behavior). The numerical simulation of a diagonal compression test and the comparison of the results with the experimental evidence are presented to validate the model.
\end{abstract}

\section{INTRODUCTION}

The performance of masonry structures under seismic actions is strongly influenced by cracking phenomena that must be considered to obtain a reliable evaluation of the structure's capacity. As experimentally observed, masonry shows signs of damage since the early stages of loading. From a microscopic point of view, when the tensile damage begins, spread and stable micro-cracks start to develop. Once the cracks growth becomes unstable, strain 
localization occurs, and macro-cracks develop. The initial phase of damage (i.e. microcracking) has an important role in the masonry modelling because affects the analysis results in terms of stiffness and ductility. At the same time, the detection of macro-cracks is fundamental to understand potential collapse mechanisms.

In general, there are two main approaches adopted for masonry modelling [1]: (i) the micromodelling approach, which usually involves the separate modelling of units, mortar and unitmortar interfaces but can involve also a lumped representation of the mortar and unit-mortar interface, with potential fracture lines localized at the interfaces; (ii) the macro-modelling approach, which does not make distinction between blocks and joints, and masonry components are smeared into an averaged continuum; the presence of cracks is introduced by changing the mechanical properties of the material. The first approach corresponds to more accurate results but computationally intensive analyses, the second one requires less computational effort but does not account for kinematic discontinuities.

In large analyses, the knowledge of the interaction between units and mortar is negligible for the global behaviour of the structure. Moreover, when the structural problem concerns rubble masonry, the distinction between blocks and joints is not always simple. All this involve the tendency to choose a macro-modelling approach in the practice-oriented analyses, excluding the possibility to realistically describe the formation and propagation of cracks.

Starting from the experimental data obtained from in-situ diagonal compression (DC) tests performed on rubble masonry walls, this study presents an original constitutive model to describe the formation and propagation of cracks in masonry, accounting for both micro and macro fractures. A mac material. The hypothesis in-plane behavior is formulation, enriched Both tension and shear cracks a formation of micro-cracks, is described as irreversible plastic deformations spread all over the

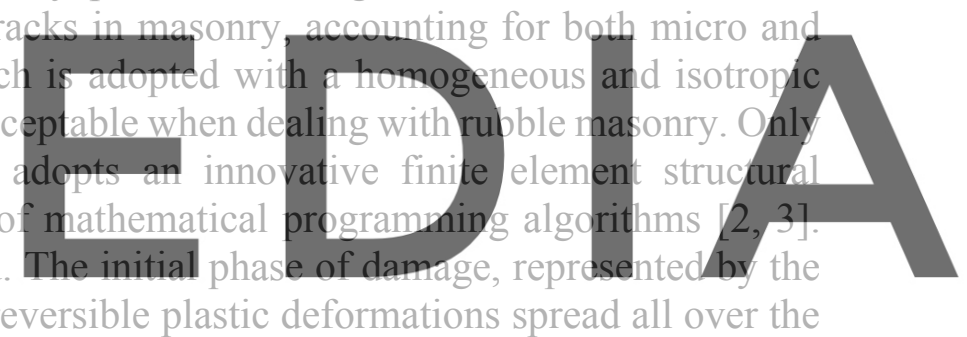

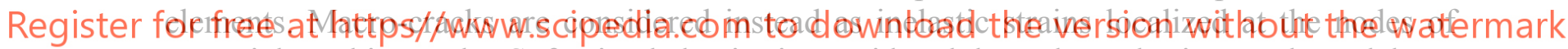
potential cracking paths. Softening behavior is considered through a cohesive crack model.

The proposed model is used to simulate a DC test to validate the formulation.

\section{EXPERIMENTAL ASPECTS}

Non-linear analysis of existing buildings, particularly for seismic loadings, requires the experimental evaluation of the post-peak degradation curve to obtain reliable analytical models. Among the available tests, the DC test is usually adopted for the determination of the shear strength of masonry walls. The in-situ version of the test is usually executed under load control on specimen cut from the existing wall by means of a circular saw or a diamond wire [4].

A special procedure has been adopted by the LPM Lab of Politecnico di Milano to execute several DC tests on the San Filippo Neri historical building in L'Aquila (Italy). The walls tested were made of rubble masonry, very common in the historical buildings of central Italy. The walls exhibit two external layers which are connected by a central infill, less predictable in terms of presence of mortar and size and type of aggregates. This implies a non-homogeneous behavior of the wall section, i.e. it is to be expected that the two external layers move with a certain independence one from the other. 

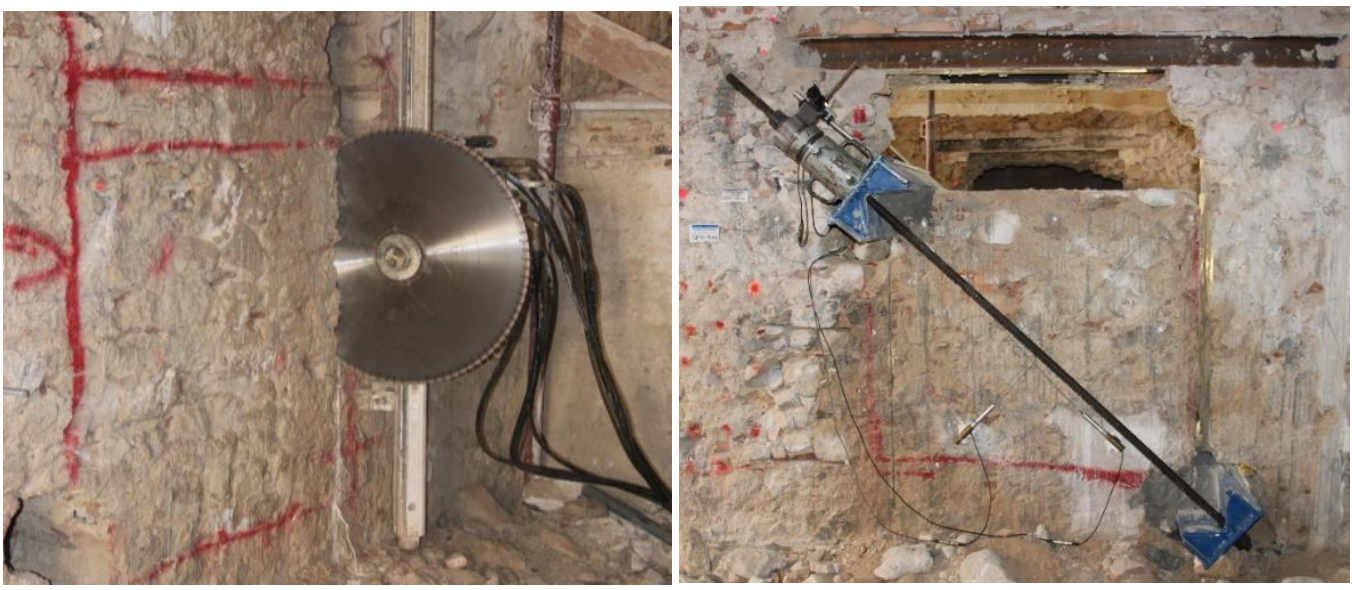

Figure 1: Left, Circular saw cutting the masonry specimen. Right, experimental equipment for the DC test.

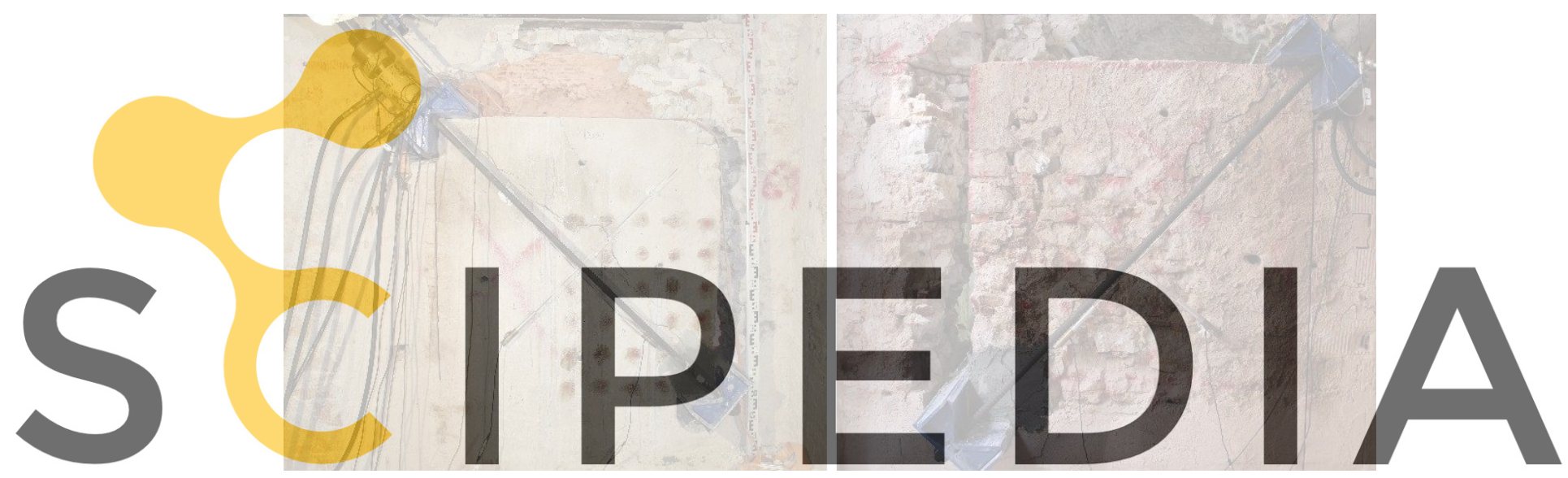

Figure 2: Cracking paths on CD01 specimens after the DC test, side 1 and 2.

Register for free at https//www.scipedia.com to download the version without the watermark
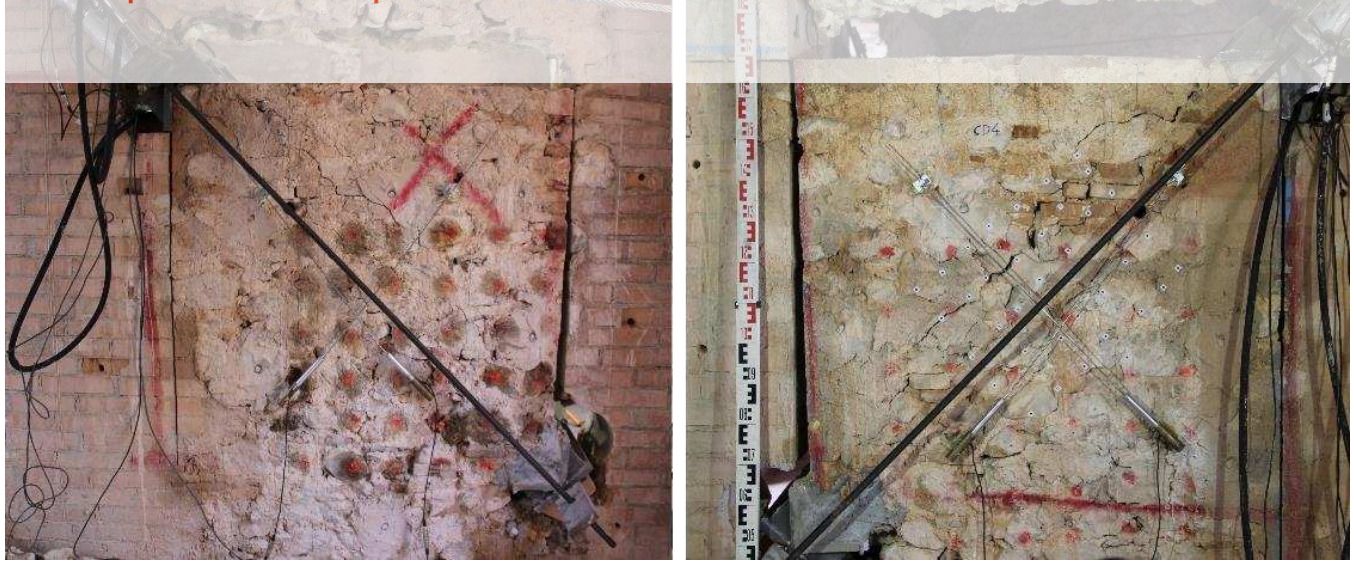

Figure 3: Cracking paths on CD04 specimens after the DC test, side 1 and 2.

Some pictures about the setting of the test are reported in Figure 1. The experimental equipment was such that the test could be performed by controlling, independently, the two jack forces acting on each masonry external layer. 
Figures 2 and 3 show the damage observed at the end of the tests in two different specimens (CD01 and CD04, both sides). The observed damage was generally represented by cracks parallel to the compressed diagonal and sliding phenomena in the vertical/horizontal direction. The crack path was mainly influenced by the location of mortar joints, which represent the weak point of the masonry assemblage.

Figure 4 shows the load vs. displacement curves. The blue and red curves represent the behavior of the two sides of each wall. Significantly different behavior of the two layers have been detected for specimen CD01, while CD04 shows a more homogenous behavior.

Two main facts come out from the observation of the curves: (i) the sharp decrease of the elastic constants in the stable part of the behavior; (ii) the irreversibility of deformations, even at the first stage of the test. It is evident that the initial phase of damage, manifested through the formation of spread micro-cracks, has a fundamental role in the masonry modelling in terms of final maximum displacement and $\sigma-\varepsilon$ plot. At the same time, the analysis of macro-cracks propagation is necessary to realistically evaluate the mechanical capacity of the structure and understand the potential collapse mechanisms. The paper will present an original constitutive model that accounts for both micro and macro-cracks.

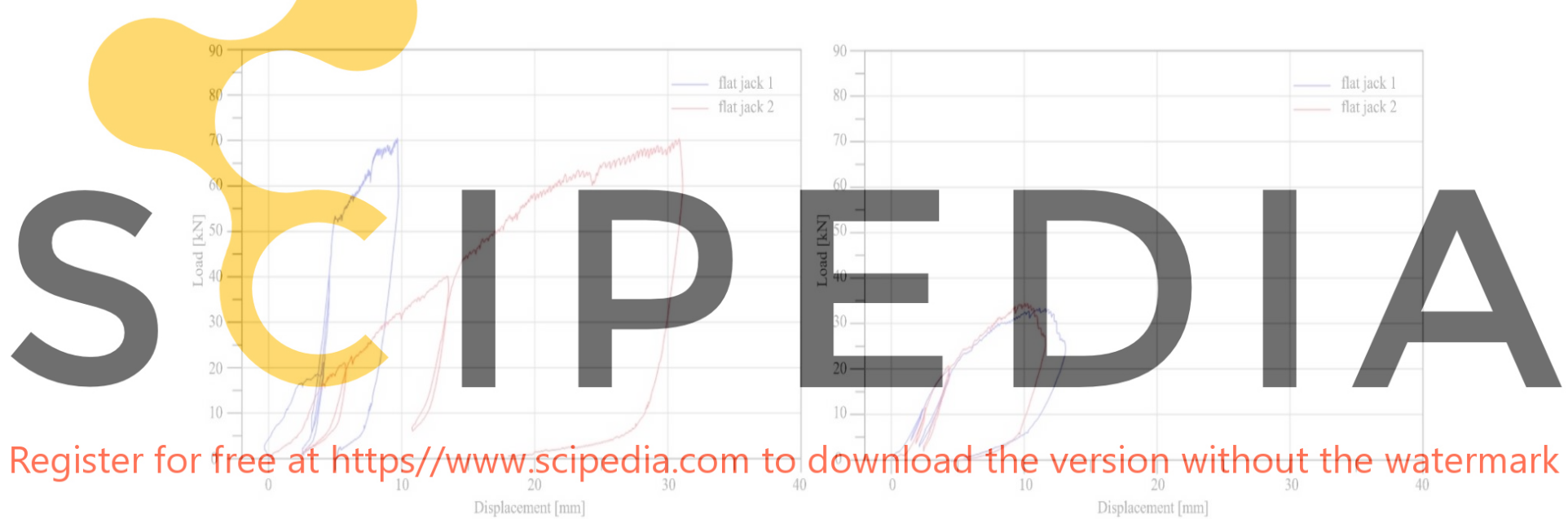

Figure 4: Load vs. displacement curves: left, specimen CD0; right, specimen CD04

\section{CONSTITUTIVE ASPECTS}

In the following formulation, tensile behavior is considered but shear can be treated analogously. The constitutive model has been subdivided in three phases: (i) undamaged linear elastic phase (Figure 5a), in which the material is considered elastic until the tension $\sigma_{\mathrm{y}}$ is reached; the value $\sigma_{\mathrm{y}}$ is a portion of the limit tension $\sigma_{\mathrm{t}}$, to be calibrated according to the experimental evidence; (ii) micro-cracking hardening phase (Figure 5b), in which micro-cracks start to propagate following an isotropic hardening law, with hardening parameter $h_{h}$, until the limit tension $\sigma_{t}$ is reached; (iii) macro-cracking softening phase (Figure 5c), in which the behavior is described by a stress-crack opening displacement constitutive law, with softening parameter $h_{s}$ and fracture energy $\mathrm{G}_{\text {f. }}$ Phases (ii) and (iii) are described in detail in the following. 


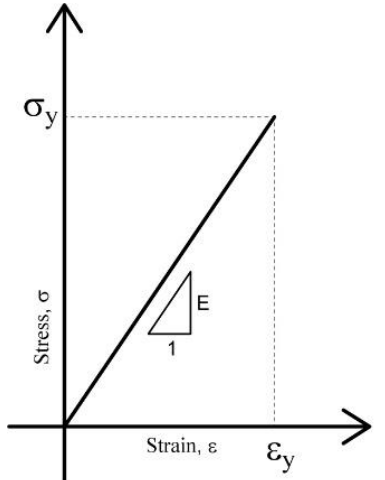

(a)

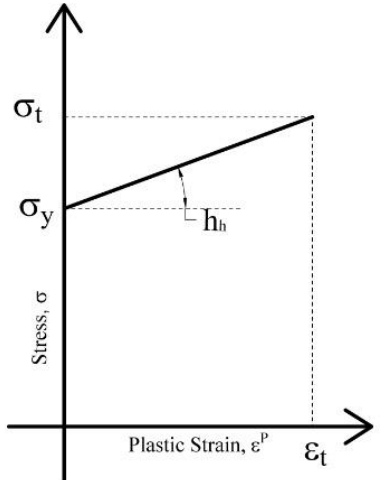

(b)

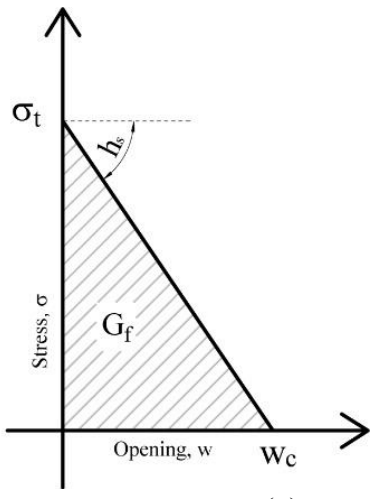

(c)

Figure 5: Constitutive law phases: (a) undamaged linear elastic phase, (b) micro-cracking hardening phase and (c) macro-cracking softening phase.

\subsection{The micro-cracking hardening phase}

The formation of micro-cracks is associated to the "stable" part of the masonry behavior in which irreversible plastic deformations start to accumulate while the load increases. The representation of plastic deformation is made according to the classical theory of plasticity, with the plastic deformation spreading in the finite elements.

Among all the available yield functions proposed in the literature, a Drucker-Prager (DP) criterion [5] has been adopted together with an associated flow-rule. The DP domain characterized by a regular and str and only two parameter

compression $\sigma_{c}$. It should be remarked that cracking phase are not $\mathrm{t}$ values to be calibrated
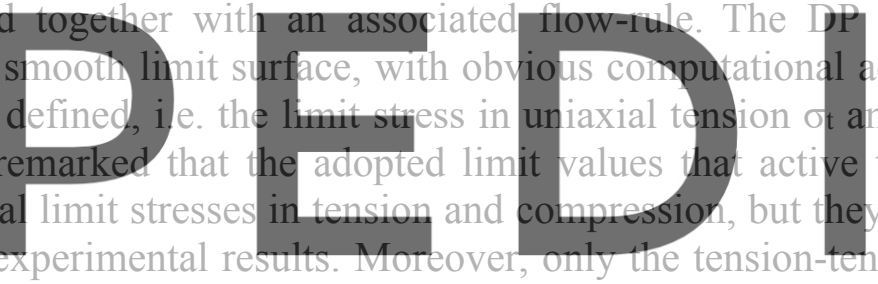

regime of the domain will be considered in this specific case.

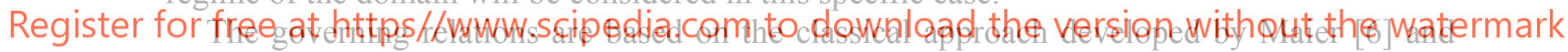

adopted by several authors $[7,8]$ where the failure surface is a piecewise linear assemblage of

linear failure surfaces. An "a posteriori" linearization procedure [9] is adopted, in which the stress points can move on the yield surface along a piecewise linearized path, lying within the bounds of a given maximum violation of the true yield function and consequently having a control on the violation of the flow-rule. The "a posteriori" linearization technique is implemented under the framework of Mathematical Programming (MP) theory and algorithms, with regard to the Linear Complementary Problem (LCP) as already proposed in [7].

Let us consider the yield surface reported in Figure 6,left. For the sake of simplicity and without loss of generality, the yield function has been limited to one parameter convex function. The elastic domain is defined by the stress points which satisfy the relation $\varphi(\sigma, \sigma 0)<0$, where $\sigma_{0}$ is the given parameter. As soon as the stress point reaches the yield surface $\varphi\left(\sigma, \sigma_{0}\right)=0$ at point A (Figure 6, right), an approximate elastic domain is defined by the stress points which satisfy the following conditions:

$$
\varphi\left(\boldsymbol{\sigma}, \sigma_{0}(1+\varepsilon)\right)<0
$$




$$
\varphi_{\mathrm{A}}=\mathbf{n}_{\mathrm{A}}^{\mathrm{T}} \boldsymbol{\sigma}_{\mathrm{A}}-\mathrm{R}_{\mathrm{A}}<0
$$

where $\mathbf{n}_{\mathbf{A}}$ is the outward unit normal to the yield surface at point $A, R_{A}$ is the distance of the plane with normal $\mathbf{n}_{\mathbf{A}}$ from the origin and $\varepsilon$ is the prescribed tolerance on the violation of the yield surface. The stress point can now move in the elastic domain or along the plane $\varphi_{\mathrm{A}}$. If the stress point violates the outer limit surface defined by (1), a back-track procedure is applied that brings the stress point on the inner curve and compute a new tangent plane.

The evolution of the yield surface is governed by an isotropic hardening law with hardening parameter $h_{h}$ (Figure 7), which represents the initial damage phase where micro-cracks develops.
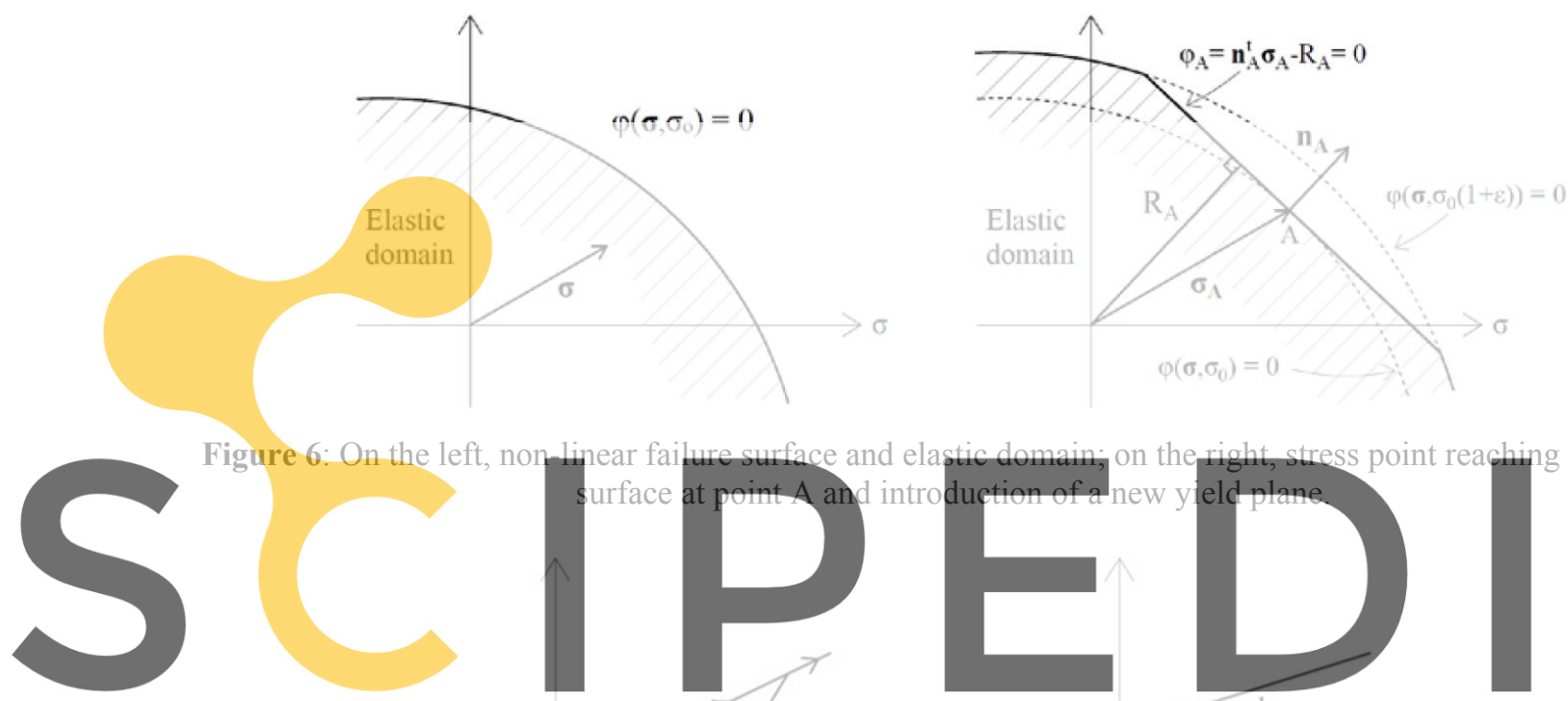

Figure 6: On the left, non-
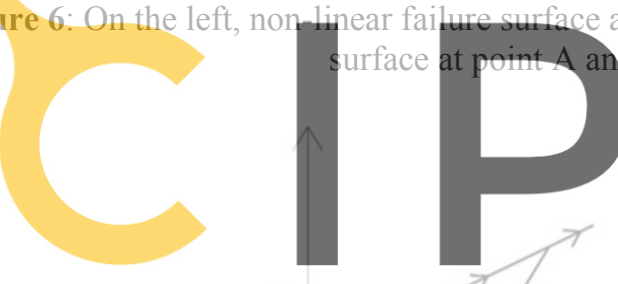

$\mathrm{r}$

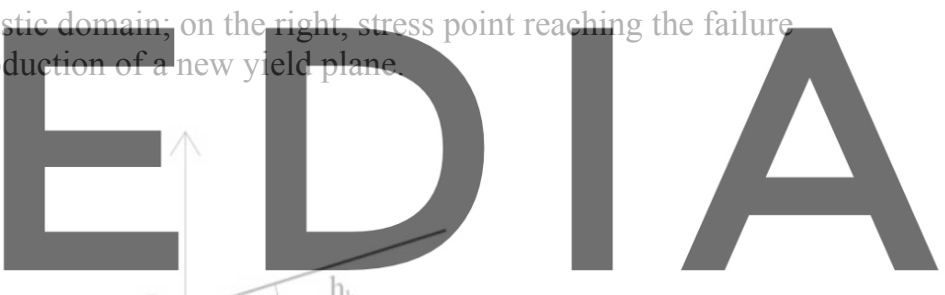

Register for free at https//www,scipedia.com to download the version without the watermark
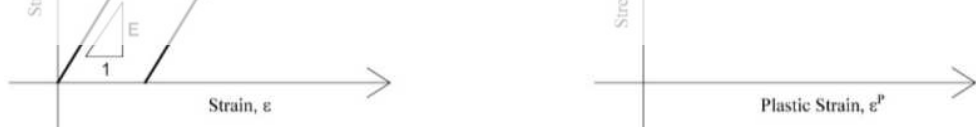

Figure 7: Elastic-plastic strain hardening model to represent the formation of micro-cracks; $h_{h}$ is the hardening parameter and $\mathrm{R}$ the resistance associated to the yield plane.

\subsection{The macro-cracking softening phase}

When the value of the limit stress in tension is reached, the macro-cracks start to propagate. At this point, in order to provide a good representation of the material behavior, strain softening must be considered. After the limit value is reached, the activated inelastic constitutive law is described as a linear softening branch, according to the cohesive crack model proposed by Hillerborg [10].

Two main assumptions are considered: (i) the crack begins to develop when the ultimate strength is achieved at one node; (ii) the material in the cracked zone is still able to transfer a force dependent on the crack opening displacement. Two parameters must be defined to properly describe the softening behavior: the limit tension $\sigma_{t}$ at which the crack starts to open 
and the critical crack width $\mathrm{w}_{\mathrm{c}}$ at which the normal stress becomes zero. The following relations link the two parameters:

$$
\begin{gathered}
\mathrm{w}_{\mathrm{c}}=\frac{2 \mathrm{G}_{\mathrm{f}}}{\sigma_{\mathrm{t}}} \\
\sigma_{\mathrm{t}}=-\mathrm{h}_{\mathrm{s}} \cdot \mathrm{w}_{\mathrm{c}}
\end{gathered}
$$

where $h_{s}$ is the softening parameter and $\mathrm{G}_{\mathrm{f}}$ is the fracture energy.

While in the micro-cracking phase the damage is spread in the elements and the plasticity control is made in terms of actual stresses, in the macro-cracking phase the plastic deformations (i.e. the cracks) are considered as localized at the nodes. The adopted failure surfaces are defined at each node and involves the nodal forces instead of the actual element stresses.

The limit tension $\sigma_{t}$ of Figure $5 c$ is then replaced by the limit traction force $R_{n}$ called resistance (Figure 8, 1eft), calculated at the generic node i as:

$$
\mathrm{R}_{\mathrm{n}}=\sigma_{\mathrm{t}} \cdot \mathrm{A}_{\mathrm{i}}
$$

where $A_{i}$ is the tributary area of the node $i$. The tributary area of a node is calculated as the product of the tributary length $\mathrm{Li}_{i}$ of the node with respect to a predefined crack direction and the thickness of the finite elements. When at one node the resistance $\mathrm{R}_{\mathrm{n}}$ is reached, the cohesive crack starts to open. The force at the node begins to decrease following the softening branch. The behavior on the softening branch is non-holonomic. If an unloading occurs, the force decreases following values of crack width and the material is cons can open and close freet

\subsection{Traction-shear interaction domain}
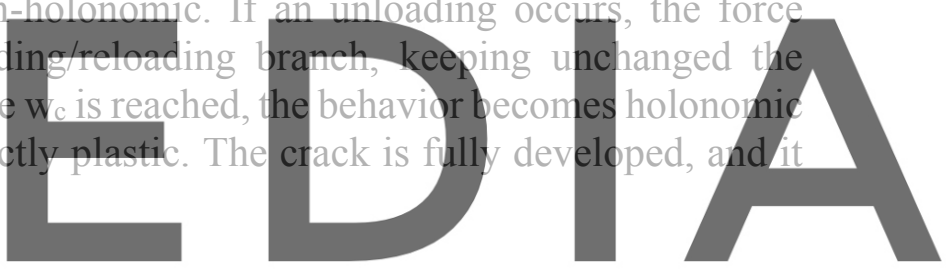

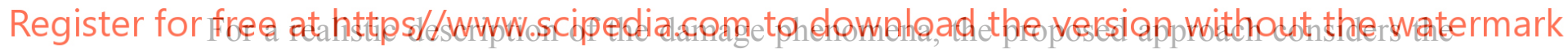

formation of both tensile cracks and shear cracks (i.e. sliding phenomena). What already presented for tension behavior can be easily extended to shear behavior without loss of generality.

At each potential cracking node belonging to a potential crack direction, two independent variables are defined: (i) w, i.e. the traction crack width, that is the normal component of the plastic deformation, and s, i.e. the shear sliding, that is the tangential component of the plastic deformation. A simple maximum stress criterion is adopted both in cases of tension and shear behavior.

The traction-shear domain for a node is represented in Figure 9 where $F_{n}$ and $F_{t}$ are respectively the traction and shear component of the nodal force and $R_{n}$ ad $R_{t}$ are the limit traction and the limit shear, calculated as the product of the tributary area of the node and the limit stress in tension $\sigma_{t}$ and shear $\tau_{\max }$, respectively. The parameters $\sigma_{t}$ and $\tau_{\max }$ are defined independently the one from the other.

The plastic deformations (i.e. cracks $\mathrm{w}$ and $\mathrm{s}$ ) develop according to the normality rule. For the sake of simplicity, no interaction between the two variables $w$ and $s$ is considered and they can be activated simultaneously only in the corner point. To make the model closer to reality, 
a traction-shear interaction may be considered by introducing a different failure domain (e.g. Mohr Coulomb criterion). This improvement will be treated in future paper.
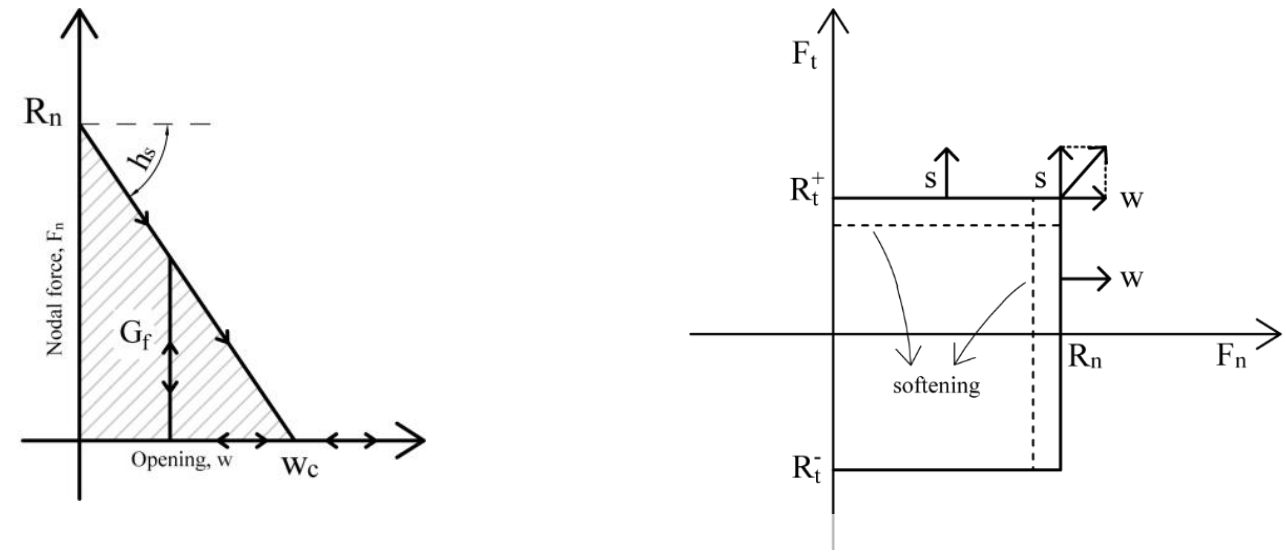

Figure 8: Left, nodal constitutive nodal law. Right, Traction-shear interaction domain.

\section{THE MACRO-CRACK MODEL}

From a numerical point of view, the macro-cracking model and its implementation in a finite element code represent an original contribution of the authors $[2,3,11]$.

Let us consider the FE model in Figure 9 (left) in which a potential cracking path $\mathrm{L}$ is identified. If a fracture develops along $\mathrm{L}$, the structure is going to be split into two, on the bottom side the line $\mathrm{L}^{+}$and the node $\mathrm{I}^{+}$and on the top side $\mathrm{L}$ on the node $I^{+}$(Fig. 9, middle), an outward unit no be defined. The vector elements $\mathrm{Q}_{j}, \mathrm{Qk}_{\mathrm{k}}$ and $\mathrm{Q}_{\mathrm{i}}$ condition at the node $\mathrm{I}^{+}$:
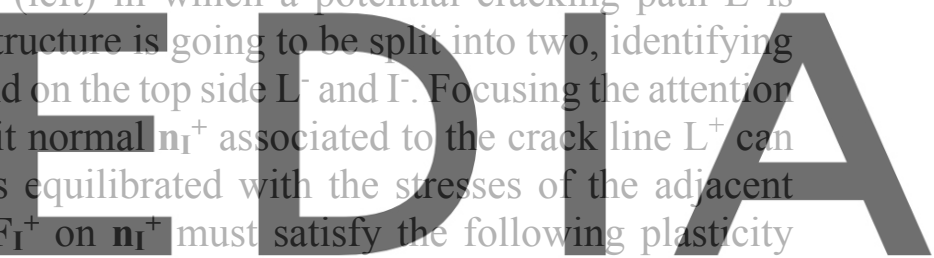

Register for free at https//www.scipedia.com tom download the version without the watermark

where $\mathrm{R}_{\mathrm{I}}{ }^{+}$is the limit tensile force, calculated as the product of the limit tension $\sigma_{t}$ times the influence area of the considered node $\mathrm{A}_{\mathrm{i}}$.
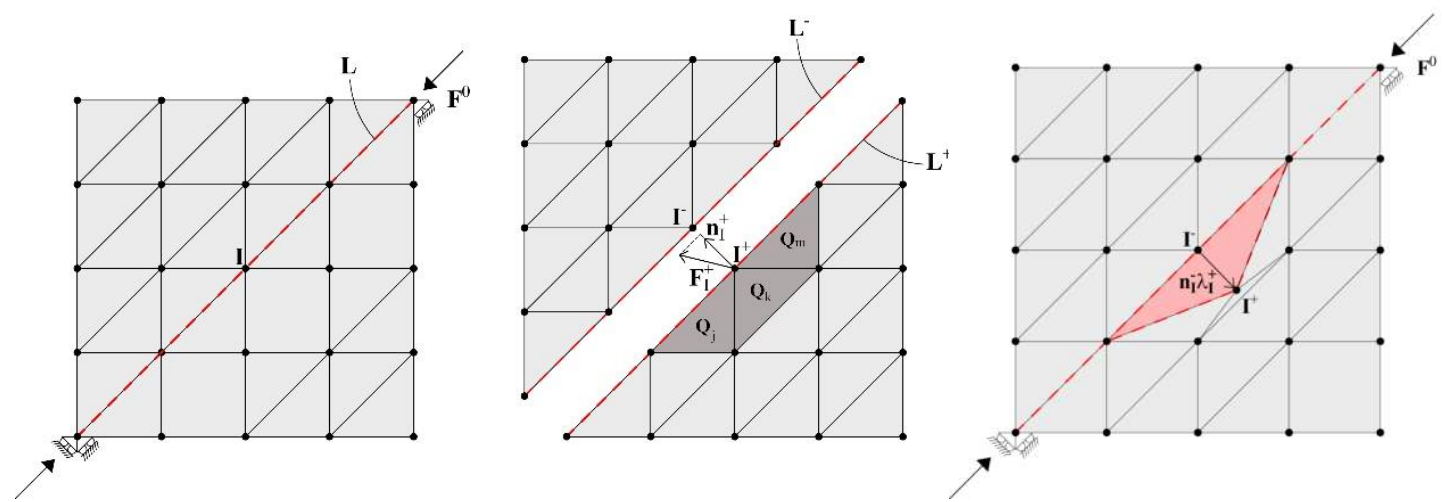

Figure 9: Left: FE model of a plane stress problem. The dashed red line L identifies a potential crack path. Middle: dal force $\mathbf{F}_{\mathbf{I}}^{+}$at $\mathrm{I}^{+}$equilibrated with $\mathbf{Q}_{\mathbf{a}}, \mathbf{Q}_{\mathbf{b}}, \mathbf{Q}_{\mathbf{c}}$. Right: Opening of the crack at node I. 
The satisfaction of the yield criteria is ensured by considering suitable imposed localized strain $\mathbf{n}_{\mathbf{I}} \lambda_{I}{ }^{+}$that represents the displacement associated to the crack opening and obeys to the normality flow rule of plasticity. When the plasticity condition is violated, the crack opens by splitting the node I into two: one belonging to $\mathrm{L}^{-}$, which remains fixed, and one belonging to $\mathrm{L}^{+}$, which moves in the direction of $\mathbf{n}_{\mathbf{I}}^{-}$opening the crack $\lambda_{\mathrm{I}}{ }^{+}$, named plastic/crack multiplier (Fig. 9, right). The crack opening affects only the bottom half structure (or better, the elements of the bottom half structure around the node $\mathrm{I}^{+}$). The same procedure can be repeated for any potential cracking node, along any possible direction.

Let us define a vector $\lambda^{+}$that collects the crack multipliers $\lambda_{I}{ }^{+}$associated to the nodes on the crack line $\mathrm{L}^{+}$, with a number of components equal to the number of potentially activable planes. A matrix $\mathbf{N}^{-}$is defined which collects, in diagonal form, the outward unit normal vectors $\mathbf{n}_{\mathbf{I}}^{-}$of each node (analogously $\mathbf{N}^{+}$can be defined for the outward unit normal vectors $\mathbf{n}_{\mathbf{I}}^{+}$). The problem to be solved is to find the elastic-plastic response of the structure under the external load $\mathbf{F}^{\mathbf{0}}$ and the prescribed inelastic crack openings $\mathbf{N}^{-} \lambda^{+}$.

According to the Colonnetti's approach [12], the elastic-plastic response of the structure is obtained as the superposition of: (i) the linear-elastic response of the structure to the given external actions; (ii) the linear-elastic response of the structure subjected to the unknown imposed strains $\mathrm{N}^{-} \lambda^{+}$. For a clear definition of response (ii), a sub-structure has to be defined made by the elements, around the considered node, lying on one side of the crack (e.g. for the node I, the dark gray elements in Figure 9, middle). Response (ii) is the superposition of: (a) the linear-elastic response of the sub-structure, considered with fixed nodes, to the imposed displacement $\mathbf{N}^{-} \lambda^{+}$, in nodal reactions are calle

Considering the potential vector $\Phi^{+} \mathrm{c}$
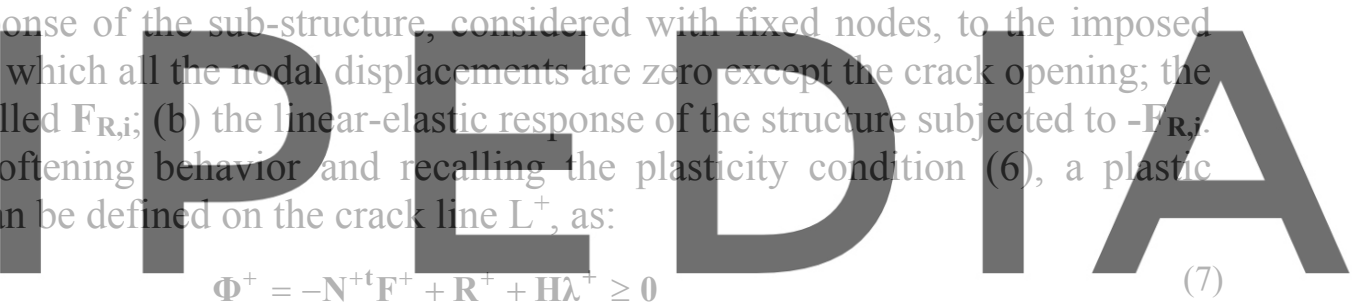

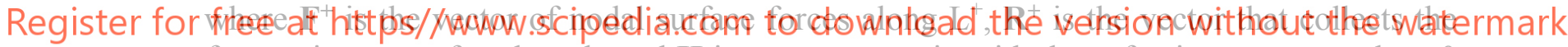
surface resistances of each node and $\mathbb{H}$ is a square matrix with the softening parameter $h_{\mathrm{s}}<0$ along the main diagonal, that has as many rows and columns as the total number of yield planes. The introduction of the matrix $\mathbf{H}$ describes how the original yield surface changes during the development of cracks. If a failure plane is activated, the corresponding potential function is zero. The governing equations of the structural problem can be expressed as a linear complementary problem (LCP) [11]:

$$
\mathbf{\Phi}^{+}=(\mathbf{A}+\mathbf{H}) \lambda^{+}-\mathbf{b}+\mathbf{R}^{+} \geq \mathbf{0}
$$

together with the so-called complementary conditions:

$$
\lambda^{+} \geq \mathbf{0}, \boldsymbol{\Phi}^{+\mathbf{T}} \lambda^{+}=0
$$

In case of irreversible inelastic deformations (i.e. non-holonomic problem), in order to capture some critical events like unloading, the conditions (9) must be expressed in rate form as follows:

$$
\dot{\lambda}^{+} \geq \mathbf{0}, \boldsymbol{\Phi}^{+\mathrm{T}} \dot{\lambda}^{+}=0
$$

Matrix $\mathbf{A}$ and vector $\mathbf{b}$ describe the behavior of the structure under imposed strains and given 
loads, respectively. The Hessian matrix A, which governs the global behavior of the structure, is symmetric and semi-definite positive. If softening is considered by the introduction of $\mathbf{H}$, the problem may become indefinite. Respect to the standard structural stiffness matrix, the matrix $\mathbf{A}$ is endowed with sophisticated mathematical features able to easily perform checks about the existence and the uniqueness of the solution $[13,14]$. Matrix $\mathbf{A}$ and vector $\mathbf{b}$ can be computed in full before the procedure is started as a series of elastic calculations which do not require the expensive computation of the inverse of the stiffness matrix $\mathbf{K}$, difficult to be determined in case of singular yield functions.

\section{COMPUTATIONAL PROCEDURES}

The LCP that governs the evolution of the system may exhibit an indefinite Hessian matrix (i.e. $\mathbf{A}+\mathbf{H})$ meaning that a multiplicity of solutions may be admitted on the equilibrium path due to softening-related bifurcation phenomena or loss of stability. Commercial finite element codes show serious difficulties in managing indefinite problem; MP algorithms have instead proved their ability to capture the whole set of possible alternative equilibrium paths and always find a solution, if it exists [14].

Solving the LCP (8) together with (9) or (10) is equivalent to solve a parametric linear complementary problem (PLCP) by introducing a scalar parameter $\alpha$ called load factor [15]:

$$
\Phi=(\mathbf{A}+\mathbf{H}) \boldsymbol{\lambda}^{+}-\boldsymbol{\alpha} \mathbf{b}+\mathbf{R}^{+} \geq 0
$$

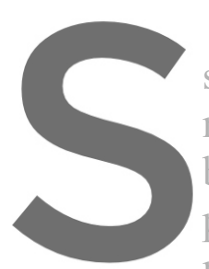

The solution of the step and the relative next inelastic point or ur by a system of linear procedure assumes basi non-holonomic constraints are
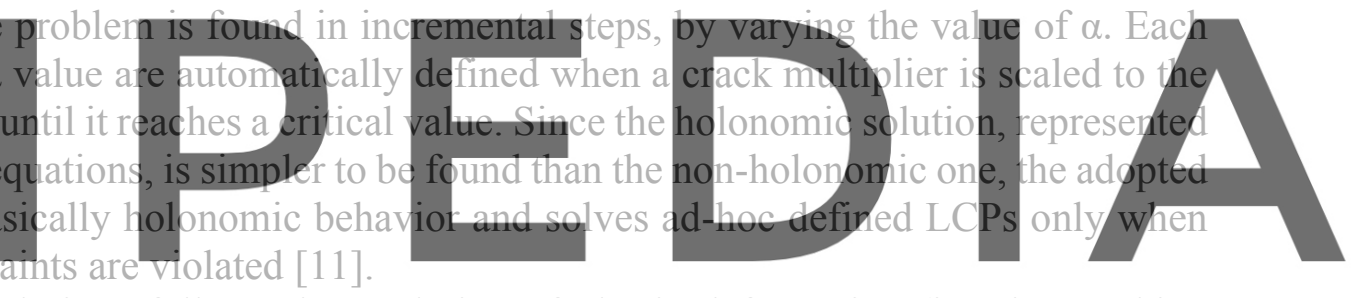

The sequence of solutions follows the evolution of plastic deformation (i.e. the cracking

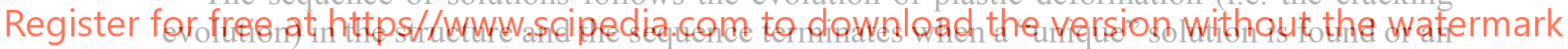
unbounded solution (i.e. collapse) is found or $\alpha$ decreases down to zero. The solution of each step is computed using a numerical code specifically developed for this purpose and which refers to the pivot theory proposed by Lemke [16].

\section{NUMERICAL SIMULATIONS}

A simulation of the in-situ diagonal compression test of sample CD04 (see paragraph 2) has been performed using the proposed constitutive model. The specimen dimensions are $1200 \mathrm{x}$ $1200 \mathrm{~mm}$ with a thickness of $420 \mathrm{~mm}$ as the panel tested in the experimental campaign. The adopted mechanical parameters are reported in Table 1. Poisson's ratio $v=0.2$ and fracture energy $\mathrm{G}_{\mathrm{F}}=0.035 \mathrm{~N} / \mathrm{mm}$ are typical values adopted in other numerical works [17]. The other parameters, i.e. Young's modulus $\mathrm{E}=1500 \mathrm{MPa}$ and tensile strength $\sigma_{\mathrm{t}}=0.12 \mathrm{MPa}$, have been quantified by calibration of the numerical and experimental results and the obtained values are consistent with typical values of historical masonry. The compression strength has been calculated as $\sigma_{\mathrm{c}}=10 \cdot \sigma_{\mathrm{t}}$. The adopted FE model is reported in Figure 12.

Figure 13 (left) shows the comparison in terms of load vs. displacement curve. The displacements of the experimental curve are calculated as the mean of the displacements of the 
two layers of wall, while the load as the sum of the loads.

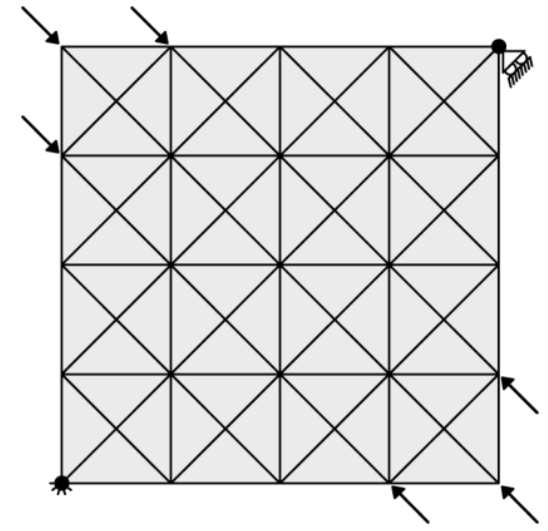

Figure 10: FE model of the masonry panel under diagonal compression
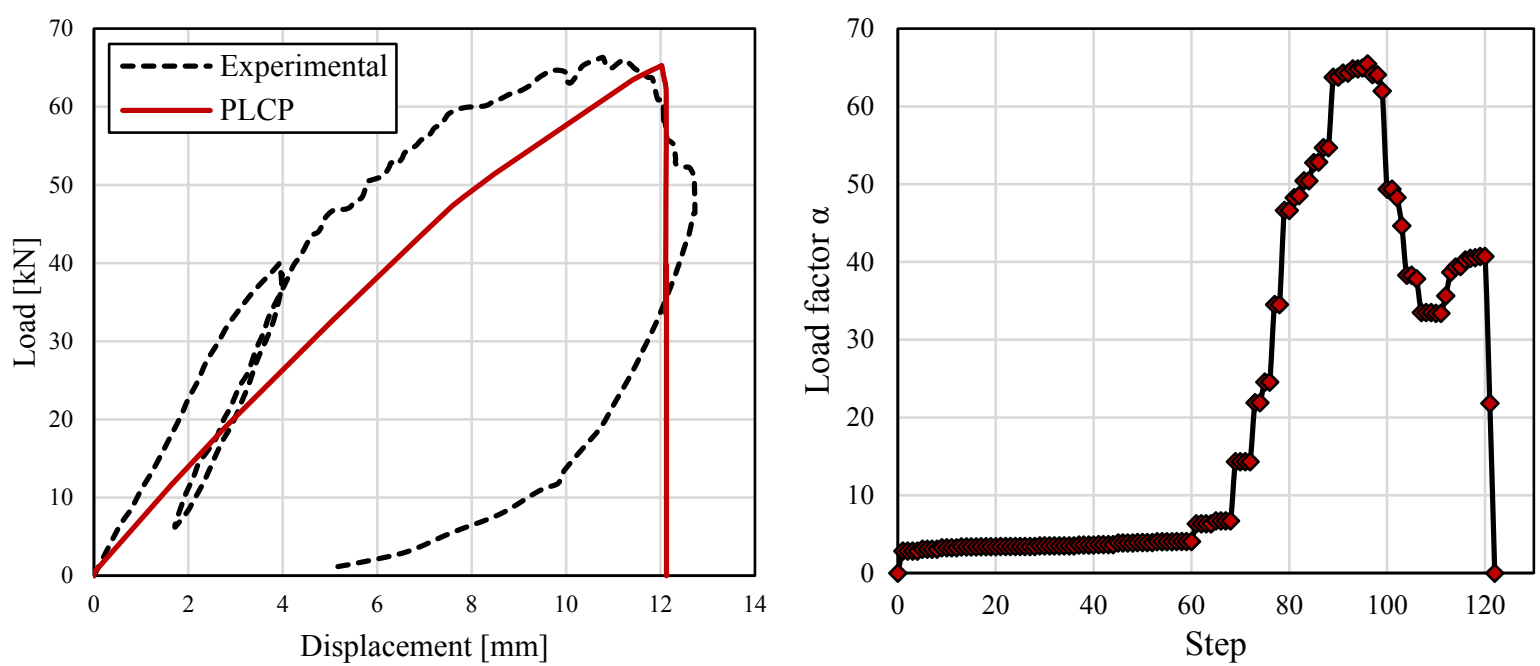

Figure 11: Left, Load vs. displacement curves comparison. Right, Load factor vs. step.
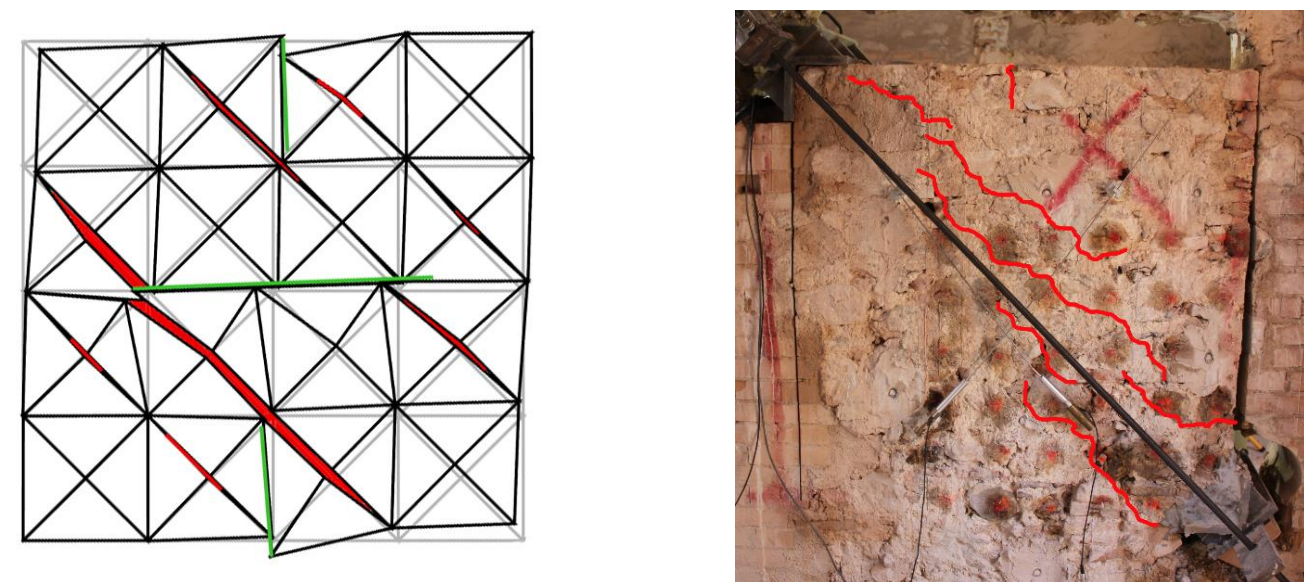

Figure 12: Left, Deformed shape at collapse with traction cracks in red and the slidings in green. Right, Experimental crack pattern. 
In the experimental curve it is possible to observe that the plasticization of the material happens already at the first steps of the test (there is no evident change of slope before the unloading/reloading and the values of stiffness are very low). The elastic limit in the numerical simulation has been set to a very low value (1\% of the failure in tension) after which the hardening branch starts, allowing a stiffness degradation able to approximate the experimental evidence. The numerical simulation catches the right value of the peak-load and the maximum displacement. The slope of the curve (PLCP), from the beginning to the peak, represents an average stiffness between the two points. A way to make the stiffness closer to the experimental one might be to modify the value of the hardening parameter during the loading history to reproduce the multi-branch hardening. However, this approach would make more complex the mathematical model.

As reported in Figure 13 (right), the load factor $\alpha$ changes during the evolutive nonholonomic analysis. The linear elastic behavior is represented by the first step. The flat section of the curve right after is the non-linear phase in which the spread plastic deformation is collected in the elements. A significant increment of the load factor is recorded when the limit tension is reached at the first node and macro-crack starts. The load factor continues to increase according to the activation of the non-holonomic softening modes at several nodes. When the critical width is reached at the first node, the problem is indefinite, the slope of the curve becomes negative and the load factor starts to decrease. Going forward with the analysis, a positive slope is recorded again meaning that the problem, from indefinite, became positivedefinite again. When the load factor becomes zero, the procedure stops.

The final deformed shape of the structure is reported in Figure 14 (left) where tension cracks have developed mainly along the compressed diagonal. Also sliding phenomena in the vertical and horizontal direction are evident. As comparison, a picture of the masonry panel after the test is reported in Figure 14 (right) and a good agreement in terms of cracks pattern is found.

\section{CONCLUSIONS}

A constitutive model to describe fracture process in masonry has been proposed. The formulation takes its cue from the evidence of experimental tests performed on historical masonry walls. The model describes the cracking phenomenon as the succession of two phases: the micro-cracking hardening phase, with cracks represented by spread plastic deformations, and the macro-cracking softening phase, with cracks represented by localized deformations at the element edges. A non-standard finite element formulation has been adopted to find the solution of the nonlinear structural problem, taking advantage of the ability of mathematical programming algorithms to manage the computational instabilities of softening materials.

The simulation of an the in-situ diagonal compression test has been performed with reference to the results of an experimental campaign conducted on a historical masonry compound in the city center of L'Aquila (Italy). Some mechanical parameters (i.e. Poisson's ratio and fracture energy) has been chosen according to typical values adopted in other scientific works. Some others (i.e. Young's modulus, tensile and compressive strength) has been quantified by calibration of the numerical and experimental results. The numerical simulation was able to catch the value of peak-load and the maximum displacement. The cracking pattern showed cracks developing mainly along the diagonal directions, together with sliding phenomena in vertical and horizontal directions, analogously to what observed after the experimental test. 
The validation test has given encouraging results; however, further research work is needed in order achieve the same results also for complex and large-scale structures.

\section{REFERENCES}

[1] Lourenço, P.B., Rots, J.G., Blaauwendraad, J., Two approaches for the analysis of masonry structures - micro and macro-modeling. Heron (1995), 40, 313-340.

[2] Scamardo, M., Franchi, A., Crespi, P., An innovative approach for the finite element modelling of masonry cracking, in: Proceedings of the 10th International Masonry Conference, The International Masonry Society, Milano (2018), pp. 132-144.

[3] Scamardo, M.A., Franchi, A., Crespi, P.G., Fe modeling of fracture in quasi-brittle material, in: Advances in Engineering Materials, Structures and Systems: Innovations, Mechanics and Applications - Proceedings of the 7th International Conference on Structural Engineering, Mechanics and Computation, 2019, (2019).

[4] Corradi, M., Borri, A., Vignoli, A., Experimental study on the determination of strength of masonry walls. Constr. Build. Mater. (2003), 17, 325-337.

[5] Drucker, D.C., Prager, W., Soil mechanics and plastic analysis or limit design. Q. Appl. Math. (1952), 10, 157-165.

[6] Capurso, M., Maier, G., Incremental elastoplastic analysis and quadratic optimization. Meccanica (1970), 5, 107-116.

[7] Cohn, M.Z., Franchi, A., Structural plasticity computer system: STRUPL. ASCE J Struct Div (1979), 105, 789-804.

[8] Tin-Loi, F., A Yield Surface Linearization Procedure in Limit Analysis*. Mech. Struct. Mach. (1990), 18, 135-149.

[9] Hodge Jr., P.G., Automatic piecewise linearization in ideal plasticity. Comput. Methods Appl. Mech. Eng. (1977), 10, 249-272.

[10] Hillerborg, A., Modeer, M., Petersson, P.E., Analysis of crack formation and crack growth in concrete by means of fracture mechanics and finite elements. Cem. Concr. Res. (1976), 6, 733-781.

[11] Scamardo, M.A., A PLCP finite element crack model for the in-plane analysis of masonry, PhD Thesis, Politecnico di Milano (2020).

[12] Colonnetti, G., Elastic equilibrium in the presence of permanent set. Q. Appl. Math. (1950), 7, 353-362.

[13] Franchi, A., Cohn, M.Z., Computer analysis of elastic-plastic structures. Comput. Methods Appl. Mech. Eng. (1980), 21, 271-294.

[14] Franchi, A., Genna, F., Corradi, L., On the determination of bifurcation and limit points. J. Eng. Mech. (1998), 124, 866-874.

[15] Patrizi, G., The equivalence of an LCP to a parametric linear program with a scalar parameter. Eur. J. Oper. Res. (1991), 51, 367-386.

[16] Lemke, C.E., On Complementary Pivot Theory, in: Mathematics of the Decision Sciences: Part 1, (1968), pp. 95-114.

[17] Milosevic, J., Lopes, M., Gago, A.S., Bento, R., Testing and modeling the diagonal tension strength of rubble stone masonry panels. Eng. Struct. (2013), 52, 581-591. 